\title{
Directed chaos in a billiard chain with transversal magnetic field
}

\author{
Holger Schanz and Manamohan Prusty \\ Max-Planck-Institut für Dynamik und Selbstorganisation und \\ Institut für Nichtlineare Dynamik der Universität Göttingen \\ Bunsenstr. 10, D-37073 Göttingen, Germany \\ E-mail: holger@chaos.gwdg.de
}

\begin{abstract}
In generic Hamiltonian systems with a mixed phase space chaotic transport may be directed and ballistic rather than diffusive. We investigate one particular model showing this behaviour, namely a spatially periodic billiard chain in which electrons move under the influence of a perpendicular magnetic field. We analyze the phase-space structure and derive an explicit expression for the chaotic transport velocity. Unlike previous studies of directed chaos our model has a parameter regime in which the dispersion of an ensemble of chaotic trajectories around its moving center of mass is essentially diffusive. We explain how in this limit the deterministic chaos reduces to a biased random walk in a billiard with a rough surface. The diffusion constant for this simplified model is calculated analytically.
\end{abstract}

\section{Introduction}

With the term directed chaos we refer to extended chaotic systems in which the timeaveraged velocity of almost all chaotic trajectories approaches for long time a non-zero constant $v_{\mathrm{ch}} \neq 0$. In contrast to the well-known deterministic chaotic diffusion, the chaotic transport is ballistic and directed in such systems, $\langle x\rangle=v_{\mathrm{ch}} t$. For 1D driven Hamiltonian systems it has been shown that directed chaos may exist if the periodic driving is such that (i) all generalized time-reversal symmetries are broken [1] and (ii) the system has a mixed phase space in which regular and chaotic dynamics coexist 2. Examples of this type have been investigated in a number of recent publications 1, 2, 3, 4, and closely related phenomena were realized experimentally with cold atoms in pulsed optical potentials [5, 6.

In the present paper we address directed chaos in a different situation which might be relevant in the context of solid-state physics. We study quasi one-dimensional billiard chains with (i) a transversal magnetic field breaking time-reversal symmetry and (ii) an asymmetric configuration of scatterers which leads to a mixed phase space. We will show that in these systems the nature of transport is entirely different for the forward and the backward direction. In one of the two directions transport is due to regular orbits skipping along a boundary of the billiard chain (waveguide) without back 
scattering. In the other direction there is strong back scattering and the dynamics is chaotic. Consequently all dynamical properties such as the average transport velocity or the superimposed spreading of a distribution of particles are different for the two transport directions. It is this special property which makes the systems we consider interesting and potentially useful for controlling nanoscale electronic transport.

We can considerably extend a preceeding study of directed chaos in magnetic billiards [7] because the specific geometry which we propose leads to a particularly simple phase-space structure. This allows for detailed analytical calculations and provides sufficient insight in order to control the transport properties of our model with a few geometric parameters. For example, we obtain an explicit expression for the chaotic transport velocity. We can also understand in some detail the velocity dispersion of the chaotic trajectories. In known examples for directed chaos [1, 2, 3, 4, this dispersion was found to be non-Gaussian, with clear signatures of Lévy walks. In contrast, we identify a parameter that can be tuned such that the dispersion becomes essentially diffusive while $v_{\mathrm{ch}} \neq 0$ remains constant. In other words, there really is a clear separation of scales between directed transport, $\langle x\rangle \sim t$, and undirected broadening of an ensemble, $\langle\Delta x\rangle \sim t^{1 / 2}$. This might be a desirable feature in applications. In this regime of biased diffusion the chaotic dynamics can be approximated by a non-deterministic random walk in a billiard with a rough boundary, although our original model is deterministic and has no disorder. Rough billiards are nowadays standard models for electron dynamics on mesoscopic scales [8, 9]. However, the system which we study here seems to be the first such model with a mixed regular-random phase space and is thus interesting also in its own right.

In the following two main sections we introduce and analyze our billiard models with directed chaos. Sec. 2 is devoted to a periodic billiard chain with transversal magnetic field in which the dynamics is deterministic, while in Sec. 3 we study a billiard with a rough surface and non-deterministic dynamics. The connection between these two models is explained in Sec. 2.3. Sec. 4 closes our paper with a summary and some concluding remarks.

\section{Periodic chain of magnetic Sinai billiards}

In Fig. 11 we show an example for an asymmetric magnetic billiard which illustrates qualitatively the effect we investigate. The system consists of a waveguide in which one wall is perfectly straight, $y_{1}(x) \equiv b$. The opposite wall is distorted periodically, $y=y_{2}(x) \geq 0$, with

$$
y_{\max }=\max _{x} y_{2}(x)<b .
$$

Specifically in our case this is achieved by a chain of semi-circular obstacles with radius $R=y_{\text {max }}$, but other geometries may lead to similar resultsł.

$\ddagger$ For example, we found similar behavior when rectangular unit cells are extended by attaching on one side a semicircle instead of cutting it out [10]. 


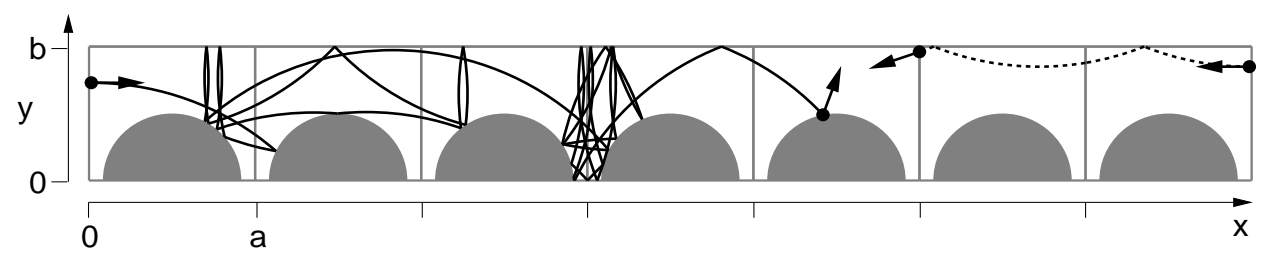

Figure 1. In a waveguide with obstacles placed only along one of the walls a transversal magnetic field leads to the coexistence of regular and chaotic trajectories. Two corresponding examples are shown with a dashed and a full line, respectively. Note that for the same magnetic field and particle charge the trajectories can be traversed only with the indicated orientation. The relevant parameters of the model are $a=1.2 b$ (spatial period), $R=0.5 b$ (radius of obstacles) and $r=2 b$ (cyclotron radius).

Energy is conserved, and we will consider the dynamics on the energy shell corresponding to the velocity $v_{0}$. For numerical calculations we use dimensionless units in which $v_{0}=b=1$, i.e., we measure velocity in units of $v_{0}$, lengths in units of $b$ and time in units of $b / v_{0}$. As a consequence, the geometric length of a trajectory is equal to the elapsed time. Due to a perpendicular magnetic field the trajectories of electrons in the waveguide consist of circular arcs with cyclotron radius $r=m v_{0} / e B$. In the following we will use the cyclotron radius to parameterize the magnetic field.

The magnetic field leads to a special set of trajectories skipping along the clean wall of the channel (dashed line in Fig. (1). It is a matter of simple geometry to describe the transport due to these regular trajectories which by our convention of the magnetic field is directed to the left (negative transport velocity). In contrast, trajectories colliding with the distorted wall of the waveguide can be chaotic such as the example shown with a full line in Fig. 1] The figure suggests that such trajectories are transporting to the right, that is opposite to the skipping ones. We will see below that this is indeed the case. This system shows directed chaos, and the transport due to the chaotic trajectories compensates for the regular skipping orbits such that no net transport results if one averages over all possible initial conditions.

\subsection{Phase space structure}

A phase-space point on the energy shell is completely described by the position $(x, y)$ in the waveguide and the angle $\varphi$ between the velocity vector and the $x$-axis. In these coordinates, the invariant measure is

$$
\mathrm{d} \mu=\mathrm{d} x \mathrm{~d} y \mathrm{~d} \varphi .
$$

In order to obtain a general impression of the dynamical scenarios supported by our model we show a Poincaré section (Fig. (2) obtained by marking $y$ and $\varphi$ whenever a trajectory crosses the boundary of a unit cell, $x=0 \bmod a$, i.e., whenever $x$ is half way between two obstacles. The two panels correspond to crossings with positive and negative transport velocity

$$
v_{x}=v_{0} \cos \varphi .
$$



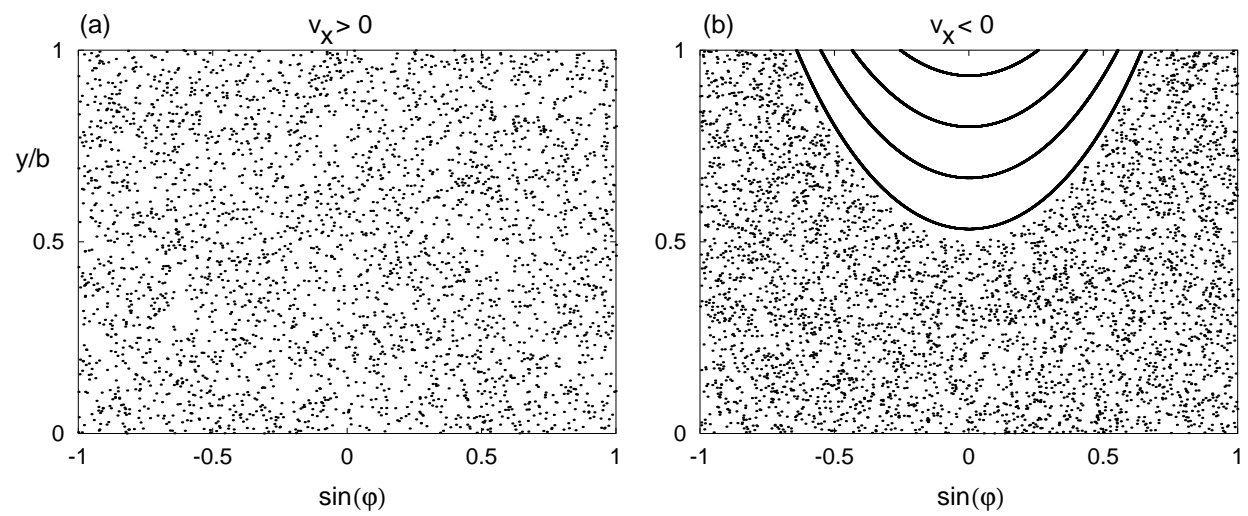

Figure 2. Phase space portrait of the system of Fig. 1 $y$ and $\varphi$ are shown for $x=0 \bmod a$ and (a) $\cos \varphi>0$, (b) $\cos \varphi<0$. The data represent one chaotic and four skipping trajectories.

Note that the invariant measure of the resulting Poincaré map is

$$
\mathrm{d} \mu_{\perp}=\mathrm{d} y \mathrm{~d} \varphi \cos \varphi .
$$

In the Poincaré section we observe a big chaotic component which contributes to both directions. In fact the randomly scattered points in Fig. $2 \mathrm{a}$ and $\mathrm{b}$ are the trace of a single chaotic trajectory, namely the one shown in Fig. 1] In addition we see in Fig. 2] also the traces of a number of skipping orbits. These trajectories have a second constant of the motion besides the energy, namely the $y$-component of the current arc's center

$$
y_{\mathrm{c}}(y, \varphi)=y-r \cos \varphi \text {. }
$$

Therefore the skipping orbits constitute a regular (integrable) component of phase space which comprises all phase-space points satisfying

$$
\cos \varphi \leq \frac{y-R}{r}-1
$$

that is all arcs whose lowest point is still above the top of the obstacles, $y_{\mathrm{c}}(y, \varphi)-r \geq$ $y_{\text {max }}$. It is clear that trajectories with such initial conditions can never be scattered. One may argue that there can exist also skipping orbits violating the condition in Eq. (6). Say the lowest point of the current arc is below $y_{\max }$ but falls into the gap between two obstacles. However, if one continues the trajectory it will finally hit one of the scatterers, at least if the longitudinal extension $\Delta x$ of the arcs is not rationally related to the spatial period $a$. Thus, exceptional skipping orbits within the chaotic component may exist but must have measure zero in phase space.

Are all orbits either chaotic or skipping? There is no reason to expect this. Typically, the boundary between a regular and a chaotic component in phase space is fractal, with hierarchies of smaller and smaller stable islands embedded into the chaotic sea. Also deep within the chaotic sea stable periodic orbits with small islands surrounding them can exist. Such islands are clearly visible for relatively small cyclotron radius, e. g. $r=1$. However, for the parameters used in Figs. 1 and 2 we were unable to detect stable islands even after increasing the resolution of the phase space portrait 
considerably. For growing cyclotron radius it must be expected that stable islands are less and less important, as for zero magnetic field $(r=\infty)$ the system reduces to the Sinai billiard (Lorentz gas) which is known to be fully hyperbolic [11. Hence, we will neglect the influence of additional stable islands and assume that for sufficiently large $r$ the phase space of our model has the simple structure which is visible in Fig. 2, All analytical calculations below will be based on this assumption.

\subsection{Average chaotic transport velocity}

We will now calculate the asymptotic chaotic transport velocity. For this purpose it is useful to restrict the dynamics to a unit cell by taking $x$ modulo $a$. After this transformation the phase-space volume of the system is finite but the transport velocity $v_{x}(t)=v_{0} \cos \varphi(t)$ is unchanged. Assuming ergodicity within the chaotic component of the unit cell, the long-time average over the transport velocity converges for almost all chaotic trajectories to the corresponding phase-space average,

$$
\begin{aligned}
v_{\mathrm{ch}} & =\lim _{t \rightarrow \infty} t^{-1} \int_{0}^{t} \mathrm{~d} t^{\prime} v_{x}\left(t^{\prime}\right) \\
& =v_{0}\langle\cos \varphi\rangle_{\mathrm{ch}} .
\end{aligned}
$$

This is illustrated in Fig. 3 As common in systems with a mixed phase space, the assumption of ergodicity within the chaotic component cannot be justified analytically. Numerical evidence in favour of it has been gathered for our model by checking that individual chaotic trajectories cover the available phase-space region uniformly.

In general there is no reason to expect $v_{\mathrm{ch}}=0$ in Eq. (7). However, an average over $\cos \varphi$ is obviously zero, if for any point $(x, y, \varphi)$ in the domain of integration also the point $(x, y, \varphi+\pi)$ with opposite velocity direction contributes. For example this is the case if the integration extends over the entire phase space of the unit cell,

$$
\langle\cos \varphi\rangle_{\mathrm{uc}}=0 .
$$

Hence, in a completely chaotic billiard we would have $v_{\mathrm{ch}}=0$, i.e., there is no directed chaos. Similarly, in any system with a single chaotic component and time-reversal symmetry there can be no directed chaos even if stable islands are present. On the other hand directed chaos can exist in fully chaotic systems with time-reversal symmetry if the phase space contains two invariant chaotic components, see [12] for an example.

In our case we can decompose the full phase space into the chaotic component and the skipping orbits. Thus we have

$$
\Omega_{\mathrm{uc}}\langle\cos \varphi\rangle_{\mathrm{uc}}=\Omega_{\mathrm{reg}}\langle\cos \varphi\rangle_{\mathrm{reg}}+\Omega_{\mathrm{ch}}\langle\cos \varphi\rangle_{\mathrm{ch}}
$$

and

$$
\Omega_{\mathrm{uc}}=\Omega_{\mathrm{reg}}+\Omega_{\mathrm{ch}},
$$

where $\Omega_{i}=\int_{i} \mathrm{~d} \mu$ denotes the phase-space volume of the set $i$. Substitution into Eq. (77) yields

$$
v_{\mathrm{ch}}=-v_{0} \frac{\Omega_{\mathrm{reg}}\langle\cos \varphi\rangle_{\mathrm{reg}}}{\Omega_{\mathrm{uc}}-\Omega_{\mathrm{reg}}}
$$




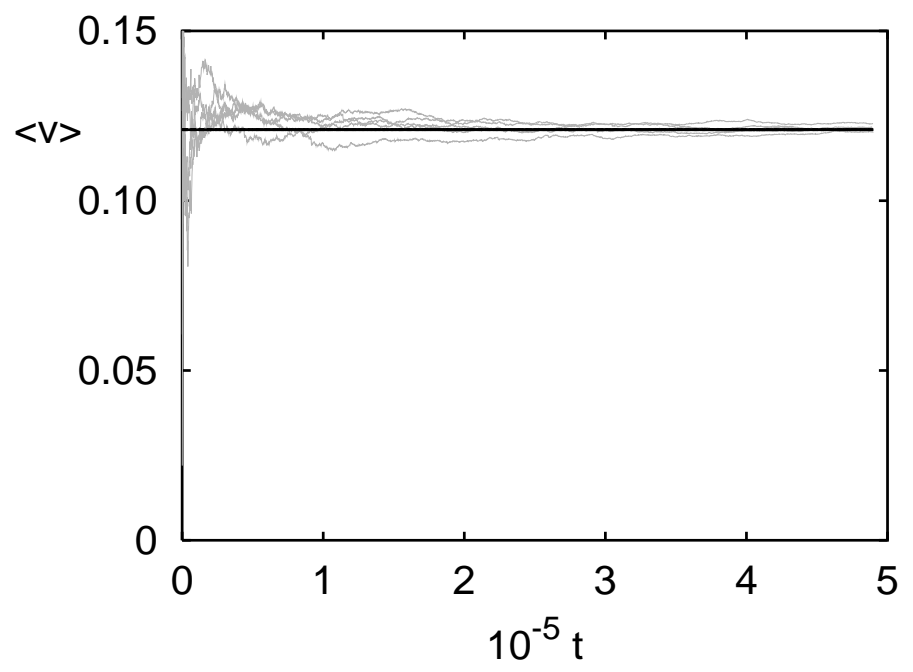

Figure 3. For the parameters of Fig. 1 the time-averaged velocity of 5 different chaotic trajectories is shown as a function of time. For all trajectories the average velocity approaches the value predicted by Eq. (17) which is indicated by the horizontal line.

which is the analogue to the sum rule derived in [2] for the chaotic transport velocity in driven 1D systems. However, in the present case we are able to calculate $v_{\text {ch }}$ explicitly. First we note that the phase-space volume of the unit cell is

$$
\Omega_{\mathrm{uc}}=2 \pi\left(a b-\frac{\pi}{2} R^{2}\right) .
$$

Next we introduce the characteristic function of the regular component which, according to Eq. (6), is given by

$$
\chi_{\mathrm{reg}}(x, y, \varphi)=\Theta\left(\frac{y-R-r}{r}-\cos \varphi\right) .
$$

The volume of the regular component is then

$$
\begin{aligned}
\Omega_{\mathrm{reg}} & =\int \mathrm{d} \mu \chi_{\mathrm{reg}}(x, y, \varphi) \\
& =a \int_{0}^{2 \pi} \mathrm{d} \varphi \int_{0}^{b} \mathrm{~d} y \Theta\left(\frac{y-R-r}{r}-\cos \varphi\right) \\
& =a \int_{\pi-\theta}^{\pi+\theta} \mathrm{d} \varphi(b-[R+r+r \cos \varphi]) \\
& =2 \operatorname{ar}(\sin \theta-\theta \cos \theta)
\end{aligned}
$$

where

$$
\theta=\arccos \left(1-\frac{b-R}{r}\right)
$$

denotes the maximum deviation of the limiting skipping orbit from the horizontal. Similarly we have

$$
\begin{aligned}
\Omega_{\mathrm{reg}}\langle\cos \varphi\rangle_{\mathrm{reg}} & =\int \mathrm{d} \mu \chi_{\mathrm{reg}}(x, y, \varphi) \cos \varphi \\
& =a \int_{\pi-\theta}^{\pi+\theta} \mathrm{d} \varphi(b-[R+r+r \cos \varphi]) \cos \varphi
\end{aligned}
$$


Directed chaos in magnetic billiards

$$
=-\operatorname{ar}(\theta-\sin \theta \cos \theta) \text {. }
$$

Substitution of these expressions into Eq. (11) finally yields

$$
v_{\mathrm{ch}}=\frac{v_{0}}{2} \frac{\theta-\sin \theta \cos \theta}{(\pi / a r)\left(a b-\frac{\pi}{2} R^{2}\right)-(\sin \theta-\theta \cos \theta)}
$$

for the chaotic transport velocity. This result is confirmed numerically in Figs. B and 4 .

For small magnetic field, when our assumption of a two-component phase space is justified best, we can approximate Eq. (17) by the leading order in the cyclotron radius. We find

$$
\theta \approx \sqrt{\frac{2(b-R)}{r}}
$$

and

$$
v_{\mathrm{ch}} \approx v_{0} \frac{4 a}{3 \Omega_{\mathrm{uc}}} \sqrt{\frac{2(b-R)^{3}}{r}} .
$$

This shows explicitly that the chaotic transport vanishes in the absence of a magnetic field $(r \rightarrow \infty)$.

\subsection{Dispersion of chaotic trajectories}

With the above results we can precisely predict the average transport velocity of many chaotic trajectories at a given moment in time, or the long-time average over a typical trajectory. However, we have no precise information about the dispersion of an ensemble, or the rate of convergence of the transport velocity to its asymptotic value. In systems with sufficiently fast decay of correlations, the dispersion of chaotic trajectories is diffusive,

$$
\left\langle\left[x(t)-v_{\mathrm{ch}} t\right]^{2}\right\rangle=D t .
$$

In this case the distribution of time-averaged velocities

$$
\bar{v}_{t}=\frac{x(t)-x(0)}{t}
$$

is for long times a Gaussian with variance $D / t$,

$$
P\left(\bar{v}_{t}\right)=\sqrt{\frac{t}{2 \pi D}} \exp \left(-[t / 2 D]\left[\bar{v}_{t}-v_{\mathrm{ch}}\right]^{2}\right) .
$$

In Fig. 4 we compare this hypothesis to the chaotic dispersion for the parameters of Fig. 1. Clear deviations from diffusive behavior are observed in the tails of the distribution and for its higher moments. After iterating $N=10^{6}$ trajectories to $t=10^{3}$ the average chaotic velocity agrees very well with the value predicted by Eq. (17). However, the distribution of time-averaged velocities is asymmetric around $v_{\mathrm{ch}}$ and, compared to a Gaussian, large deviations from the mean value are enhanced. For the considered time interval the variance of the distribution $(+)$ is fitted well by the diffusive

prediction $D / t$. However, with increasing $n$ the higher moments $\left\langle\left(v-v_{\mathrm{ch}}\right)^{n}\right\rangle$ of the velocity distribution deviate more and more from the corresponding time-dependent 

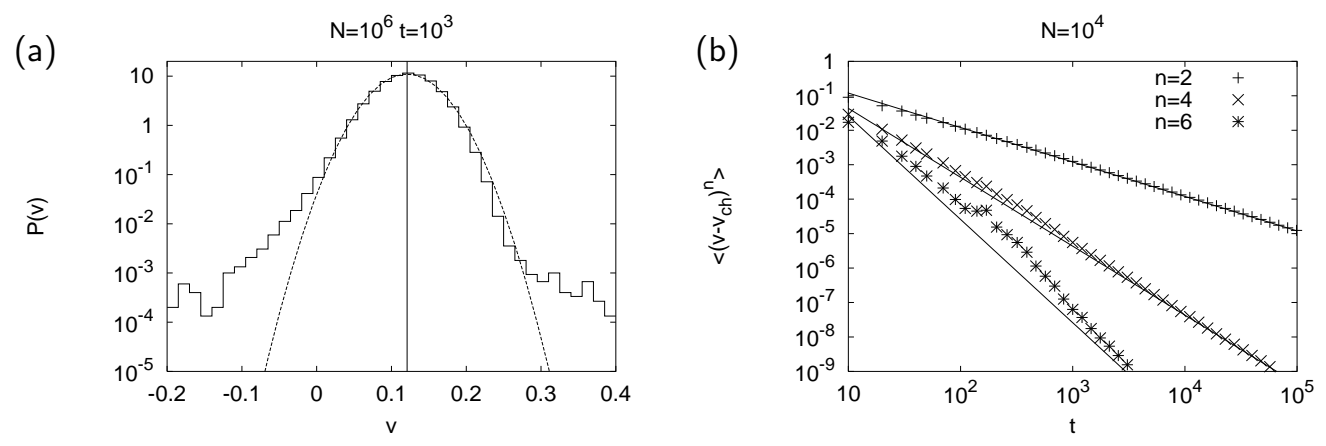

Figure 4. For the model of Fig. 1 the dispersion of chaotic transport velocities is analyzed. (a) $N=10^{6}$ trajectories have been iterated up to $t=10^{3}$. The resulting distribution of average velocities is displayed as a histogram and compared to a Gaussian with the same mean and variance (dotted line). The prediction of Eq. (17) for the average velocity is indicated by a vertical line. The numerical mean value of the distribution differs from that by $1.5 \times 10^{-5}$. (b) shows some higher moments $\left\langle\left(\bar{v}-v_{\mathrm{ch}}\right)^{n}\right\rangle$ of the distribution as a function of time. The straight lines indicate the corresponding moments of a diffusively spreading Gaussian with $D=1.2$.
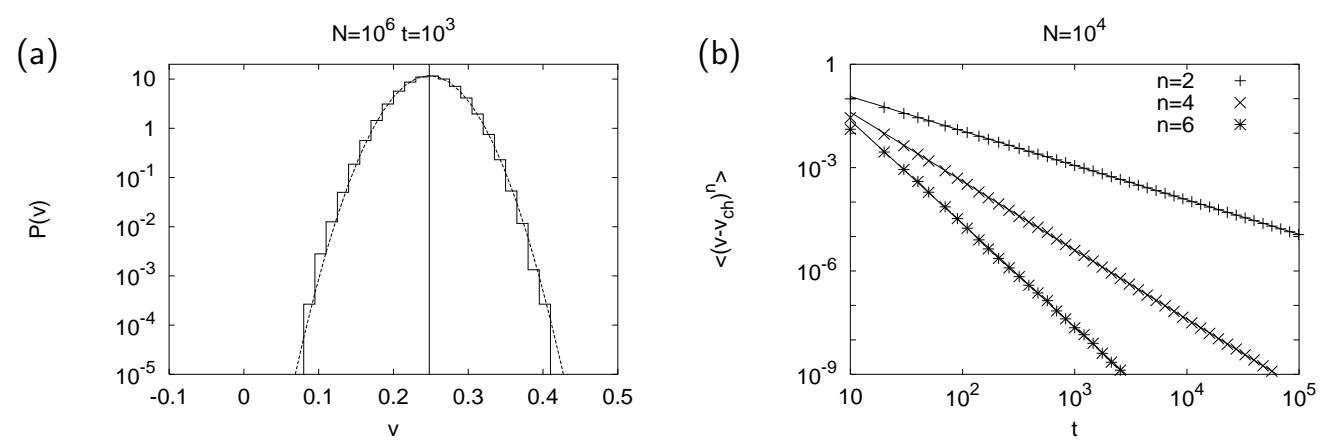

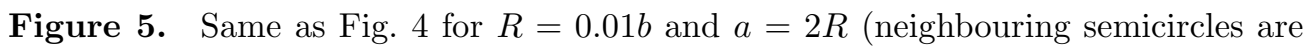
touching). The fitted diffusion constant is $D=1.2$

Gaussian $(\times, *$ vs full lines). This is no surprise as in systems with a mixed phase space anomalous diffusion is typical. Indeed, in previous studies of directed chaos in such systems the observed velocity distributions were always non-Gaussian [1, 2, 3, 4. They displayed traces of Lévy walks and anomalous diffusion which were even stronger

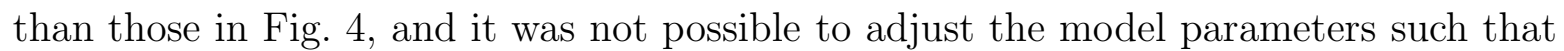
directed transport coexists with normal diffusion.

Therefore it is quite remarkable that in our model a slightly modified geometry leads to a velocity distribution which numerically could not be distinguished from a Gaussian. In Fig. [5 the analysis of Fig. [4 is repeated for a system in which (i) the semicircular obstacles are much smaller than in Fig. 1 and (ii) neighbouring scatterers are tangent rather than separated by a gap (see Fig. Th).

This latter modification removes marginally stable periodic orbits which hit the lower wall within the straight sections of the boundary only (Fig. G). Up to a certain limit it is possible to translate such orbits along the channel without changing their shape. Nevertheless they form a set of measure zero in phase space since the angle of 


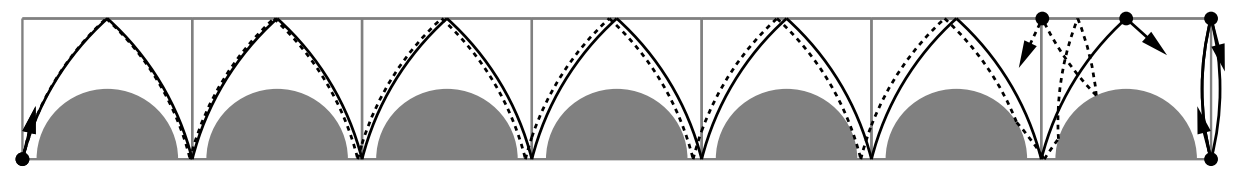

Figure 6. The full lines show two marginally stable periodic orbits (one of them is periodic up to translation by one unit-cell length). The dotted line is a non-periodic chaotic trajectory which remains in the vicinity of a marginally stable orbit for a very long period of time.

the trajectory cannot be varied without destroying the orbit (dotted line in Fig. 6). It is known that marginally stable trajectories are a source of non-exponential decay of correlations. They lead to anomalous diffusion even in completely chaotic systems like the Sinai or the stadium billiard. In our system generic chaotic trajectories can remain in their vicinity for a long time and during these episodes keep the transport velocity substantially above or below the average (e. g. $v \approx 0.5$ for the dotted line in Fig. [6] while $\left.v_{\mathrm{ch}}=0.12\right)$. Therefore it is quite natural that the chaotic dispersion shows much less anomalous diffusion when the specific geometry prevents the existence of marginally stable trajectories.

In contrast, it is not immediately clear why reducing the radius of the scatterers should further suppress anomalous diffusion in our model. In order to find an intuitive explanation for this numerical fact we consider now a different Poincaré section defined by the intersections of a trajectory with the line $y=R$. Note that regular skipping orbits do not at all intersect this line. In analogy to Eq. (4) the invariant measure restricted to the surface of section is now

$$
\mathrm{d} \mu_{\|}=\mathrm{d} x \mathrm{~d} \varphi \sin \varphi=\mathrm{d} x \mathrm{~d} c
$$

with $c=\cos \varphi$. Hence, a typical chaotic trajectory is represented in this Poincaré section by points $\left(\xi_{n}, c_{n}\right)$ which uniformly cover the area $[0,1] \times[-1,1] . \quad \xi_{n}=\left(x_{n} / a\right) \bmod 1$ denotes here the position of the intersection relative to the unit cell. Following a transition across $y=R$ from below, $\left(\xi_{n-1}, c_{n-1}\right)$, there will always be an intersection from above which is denoted by $\left(\xi_{n}^{\prime}, c_{n}^{\prime}\right)$. Geometry requires $c_{n}^{\prime}=c_{n-1}$.

For small obstacle radius transport is mainly due to the long segments of the trajectory above $y=R$ (Fig. [7). Each segment is either a single circular arc or a combination of two arcs which are identical up to a reflection. Segment $n$ is entirely characterized by the value of $c_{n}$. Therefore the statistics of the $c_{n}$ determine the transport properties. In particular, the velocity dispersion will be diffusive if correlations between consecutive angles decay sufficiently fast. This argument will be made more explicit in Sec. 3] below. Fig. 75 shows that for sufficiently small obstacle radius $\left\langle c_{0} c_{n}\right\rangle$ indeed decays very fast as a function of $n$ (exponentially fast to a good approximation). This observation can be understood as follows: As $R \rightarrow 0$, a segment $\left(\xi_{n-1}, c_{n-1}\right) \rightarrow\left(\xi_{n}^{\prime}, c_{n}^{\prime}\right)$ traverses more and more unit cells between its end points. As a consequence, one expects an extremely rapid variation of $\xi_{n}^{\prime}$ as a function of $c_{n-1}$. This 

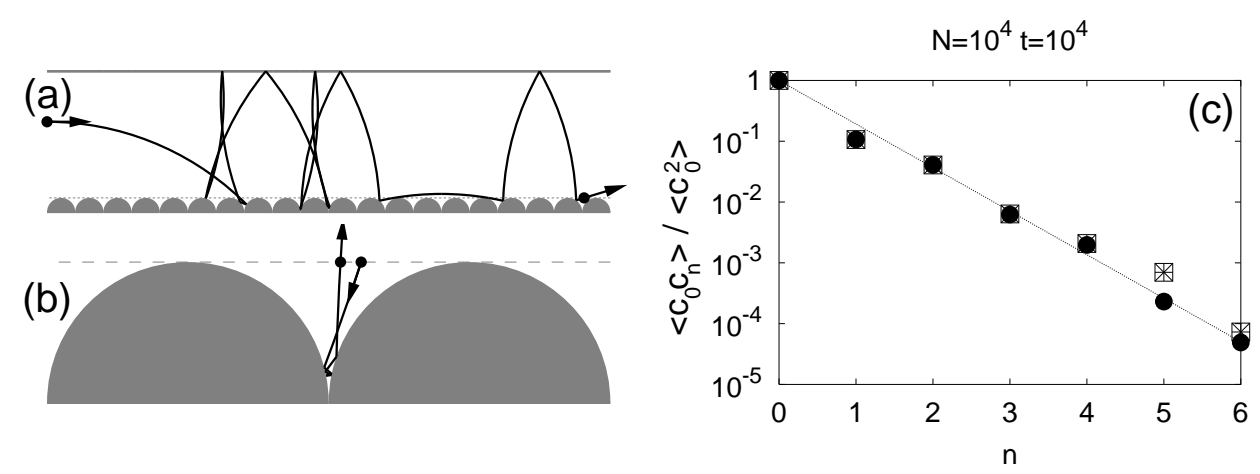

Figure 7. (a) A piece of a chaotic trajectory is shown for $R=0.1 b$ and $a=2 R$. (b) One particular scattering of this trajectory from the lower billiard boundary is magnified. Note that on this scale the curvature of the trajectory due to the magnetic field is hardly visible. (c) The normalized correlation between consecutive intersection angles of the segments with the line $y=R$ is shown with stars for the model of (a), with empty squares for $R=0.01 b$ and and with black dots for the same $R$ but randomized $\xi_{n}$ (see text). The straight line shows the fitted exponential $\exp (-1.65 n)$.

suggests to replace $\xi_{n}^{\prime}$ by a random variable. Fig. [7 confirms that this approximation is very good for the parameters of Fig. [7 or even smaller obstacle radius $R$. Note that the argument leading to this randomization does not depend on the value of the cyclotron radius $r$. In particular, it remains valid also in the absence of a magnetic field, $r=\infty$.

Next we consider the dynamics in the vicinity of the lower boundary, i.e., for $y<R$. For small obstacle radius it is practically identical to that for $r=\infty$ since the ratio $2 R / r$ between the size of the unit cell and the (constant) radius of curvature of the trajectory vanishes as $R \rightarrow 0$ (Fig. [7b). Hence the resulting mapping $\left(\xi_{n}, c_{n}\right) \rightarrow\left(\xi_{n}^{\prime}, c_{n}^{\prime}\right)$ may be approximated by a Sinai billiard without magnetic field. The latter is known to be hyperbolic [11] and shows exponential mixing if no marginally stable orbits are present. As the correlations should not be enhanced by the additional randomization of $\xi$ we finally understand why $\left\langle c_{0} c_{n}\right\rangle$ decays exponentially for small obstacle radius $R \ll r$, leading to a diffusive velocity dispersion.

\section{A waveguide with one rough boundary}

The results of the previous section suggest that for small obstacle radius $R$ the detailed dynamics inside the lower boundary layer of the waveguide is irrelevant. This holds at least as long as the dynamics therein is chaotic. In this case the essential physical properties of our model are not affected if we replace the boundary layer by an idealized rough surface and describe the scattering at the lower wall probabilistically. In the present section we follow this approach and obtain in this way a better analytical understanding. For the sake of simplicity we will continue to refer to trajectories which hit the lower wall as chaotic although the dynamics is not deterministic anymore. This is justified since we have just explained how random scattering may arise from 

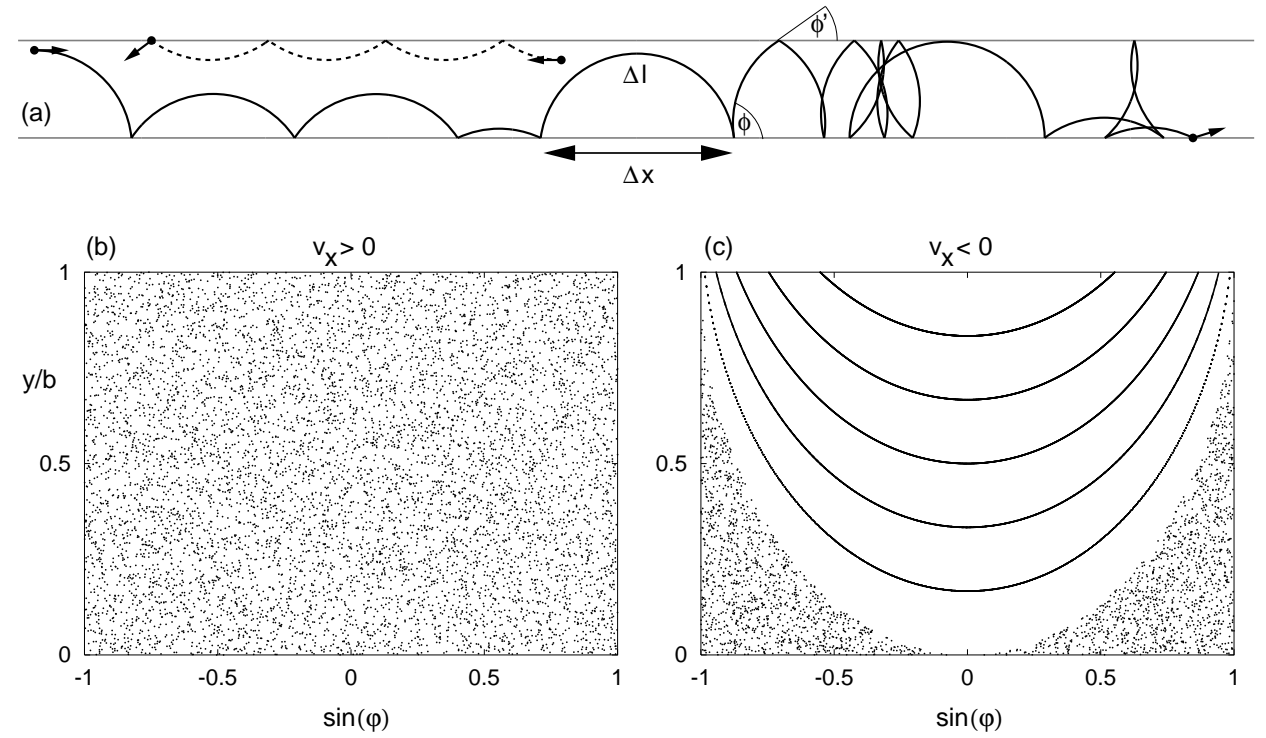

Figure 8. (a) Typical electron trajectories (dashed line: regular, full line: chaotic) for a waveguide with a perpendicular magnetic field $(r=5 b / 3)$. The lower wall is perfectly rough $(\alpha=0)$ such that the angles before and after a reflection are completely uncorrelated. (b) and (c) show a phase space portrait of this system for $r=b$.

a deterministic chaotic system in a certain limit. Nevertheless there are also other mechanisms such as thermal or quantum fluctuations which may lead to the same probabilistic model.

In order to define our simplified model we must prescribe the probability for a trajectory to leave the lower boundary at an angle $0 \leq \phi \leq \pi$. Consistency with the invariant measure $d \mu_{\|}$, Eq. (23), requires

$$
P(\phi)=\frac{\sin \phi}{2} \quad(0 \leq \phi \leq \pi) .
$$

In terms of $c=\cos \phi$ this amounts to

$$
P(c)=\frac{1}{2} \quad(-1 \leq c \leq+1)
$$

for the probability density averaged over all chaotic trajectories. We account for possible correlations between the segments of a trajectory by introducing a probability $\alpha$ for a specular reflection at the lower boundary. The probability density for $c_{n}$ is then

$$
P\left(c_{n}\right)=\alpha \delta\left(c_{n}-c_{n-1}\right)+\frac{1-\alpha}{2} .
$$

While for $\alpha=0$ there are no correlations between consecutive values of $c_{n}$

$$
\begin{aligned}
\left\langle c_{0} c_{n}\right\rangle & =\delta_{n, 0} \int_{-1}^{+1} \mathrm{~d} c P(c) c^{2} \\
& =\frac{\delta_{n, 0}}{3} \quad(\alpha=0),
\end{aligned}
$$

the possibility of specular reflections leads to exponentially decaying correlations

$$
\left\langle c_{0} c_{n}\right\rangle=\frac{\alpha^{n}}{3} \quad(\alpha \neq 0) .
$$


For each segment of a chaotic trajectory we can express the horizontal distance between its endpoints as

$$
\Delta x(\phi)=2 r\left(\sin \phi-\sin \phi^{\prime}\right) .
$$

On the other hand the total length of a segment is

$$
\Delta l(\phi)=2 r\left(\phi-\phi^{\prime}\right) .
$$

Here $\phi$ and $\phi^{\prime}$ denote the angles of intersection with the lower and the upper wall of the channel, respectively (see Fig. [ 8 a). In terms of the former, the latter is given by

$$
\phi^{\prime}=\left\{\begin{array}{ll}
\arccos \left(\cos \phi+\frac{b}{r}\right) & \left(\cos \phi \leq 1-\frac{b}{r}\right) \\
0 & \left(\cos \phi>1-\frac{b}{r}\right)
\end{array},\right.
$$

where the second line extends the definition of $\phi^{\prime}$ to those segments of a trajectory which do not reach the upper wall.

With the help of the angle $\phi$ we can give an alternative parameterization of the chaotic component of phase space. Instead of $(x, y, \varphi)$ we can refer to one of its points by the coordinates $(x, \phi, l)$. As mentioned above, $\phi$ is the inclination angle of the segment at its initial point, and $0 \leq l \leq \Delta l(\phi)$ is the path length measured from there. Formally, the transformation between $(\phi, l)$ and $(\varphi, y)$ is given by

$$
\begin{aligned}
& \varphi(\phi, l)= \begin{cases}\phi-\frac{l}{r} & l \leq \frac{\Delta l(\phi)}{2} \\
-\varphi(\phi, \Delta l-l) & l>\frac{\Delta l(\phi)}{2}\end{cases} \\
& y(\phi, l)=r \cos \varphi(\phi, l)-r \cos \phi,
\end{aligned}
$$

where the second line of Eq. (32) expresses the reflection symmetry of the trajectory segments. In terms of $\phi$ and $l$ we can express arbitrary averages over the chaotic phase space component as

$$
\begin{aligned}
\langle\ldots\rangle_{\mathrm{ch}} & =\frac{\int_{0}^{\pi} \mathrm{d} \phi P(\phi) \int_{0}^{\Delta l(\phi)} \mathrm{d} l(\ldots)}{\int_{0}^{\pi} \mathrm{d} \phi P(\phi) \int_{0}^{\Delta l(\phi)} \mathrm{d} l} . \\
& =\langle\Delta l\rangle_{\phi}^{-1} \int_{0}^{\pi} \mathrm{d} \phi P(\phi) \int_{0}^{\Delta l(\phi)} \mathrm{d} l(\ldots),
\end{aligned}
$$

where $\langle\ldots\rangle_{\phi}$ is the average over the probability density $P(\phi)$. Eq. (34) will be applied in the following sections.

It is useful to note that Eqs. (29) and (30) allow to understand the chaotic transport as random walk along the $x$-axis without any reference to the transversal motion. The discrete steps of this random walk are $\Delta x(\phi)$ and the corresponding time increments are $\Delta l(\phi) / v_{0}$. Step $n$ is chosen according to the probability density given in Eq. (26).

\subsection{Average chaotic transport velocity}

We will now give an alternative derivation for the chaotic transport velocity in the case $R=0$. We can apply Eq. (34) to Eq. (77) and simplify the result using the identity

$$
\int_{0}^{\Delta l(\phi)} \mathrm{d} l \cos \varphi(\phi, l)=\Delta x(\phi)
$$


which follows from Eq. (32) and should also be obvious from the geometrical meaning of $\Delta x$ and $\Delta l$. In this way we obtain

$$
\begin{aligned}
v_{\text {ch }} & =v_{0}\langle\Delta l\rangle_{\phi}^{-1} \int_{0}^{\pi} \mathrm{d} \phi P(\phi) \int_{0}^{\Delta l(\phi)} \mathrm{d} l \cos \varphi \\
& =v_{0}\langle\Delta l\rangle_{\phi}^{-1} \int_{0}^{\pi} \mathrm{d} \phi P(\phi) \Delta x(\phi) \\
& =v_{0} \frac{\langle\Delta x\rangle_{\phi}}{\langle\Delta l\rangle_{\phi}} .
\end{aligned}
$$

This representation is very intuitive from the random-walk point of view: a long trajectory consists of $N \rightarrow \infty$ steps distributed according to $P(\phi)$. Therfore the distance between the end points of the trajectory is $\Delta X=N\langle\Delta x\rangle_{\phi}$ while the total time increment is $\Delta T=N\langle\Delta l\rangle_{\phi} / v_{0}$. The average longitudinal component of the velocity is then $\Delta X / \Delta T$ which is equivalent to Eq. (36). Explicit averaging over $\phi$ yields

$$
\begin{aligned}
\langle\Delta x\rangle_{\phi} & =r \int_{0}^{\pi} \mathrm{d} \phi \sin \phi\left(\sin \phi-\sin \phi^{\prime}\right) \\
& \left.=r \int_{-1}^{+1} \mathrm{~d} c \sqrt{1-c^{2}}-r \int_{-1}^{1-b / r} \mathrm{~d} c \sqrt{1-[c+b / r]^{2}}\right) \\
& =\frac{\pi}{2} r-\frac{r}{4}\left[\pi+2\left(1-\frac{b}{r}\right) \sqrt{\frac{b}{r}\left(2-\frac{b}{r}\right)}-2 \arcsin \left(\frac{b}{r}-1\right)\right] \\
& =\frac{r}{2}(\theta-\sin \theta \cos \theta) .
\end{aligned}
$$

In agreement with Eq. (15) we have set $\cos \theta=1-b / r$ in order to obtain the last line. For the average length of a segment we have

$$
\begin{aligned}
\langle\Delta l\rangle_{\phi} & =r \int_{0}^{\pi} \mathrm{d} \phi \sin \phi\left(\phi-\phi^{\prime}\right) \\
& =r \int_{-1}^{+1} \mathrm{~d} c \arccos (c)-r \int_{-1}^{1-b / r} \mathrm{~d} c \arccos (c+b / r) \\
& =\pi r-\frac{r}{2}\left[\left(1-\frac{b}{r}\right)\left(\pi-2 \arcsin \left(\frac{b}{r}-1\right)\right)+2 \sqrt{\frac{b}{r}\left(2-\frac{b}{r}\right)}\right] \\
& =r\left[\pi \frac{b}{r}-(\sin \theta-\theta \cos \theta)\right] .
\end{aligned}
$$

Combining Eqs. (36)-(38) we find the expected result, namely Eq. (17) with $R=0$ (and arbitrary $a$ )

$$
\begin{aligned}
v_{\mathrm{ch}} & =\frac{v_{0}}{2} \frac{\theta-\sin \theta \cos \theta}{(\pi b / r)-(\sin \theta-\theta \cos \theta)} \\
& \approx \frac{2 v_{0}}{3 \pi} \sqrt{\frac{2 b}{r}} \quad(r \rightarrow \infty) .
\end{aligned}
$$

Fig. 9 shows Eq. (39) as a function of the cyclotron radius. We observe that for small $r$ the properties of the system change qualitatively. In particular the chaotic transport velocity is constant, $v_{\mathrm{ch}}=v_{0} / 2$ for $r \leq b / 2$. In this regime a third phase 


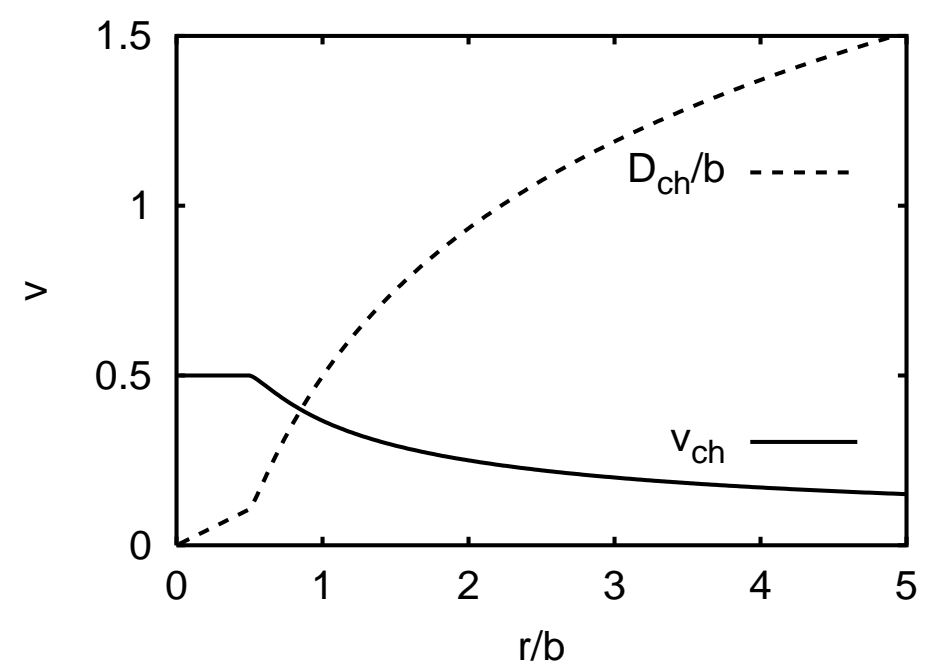

Figure 9. Dependence of the chaotic transport velocity and the diffusion constant on the cyclotron radius $r$ for random scattering from the lower wall $(\alpha=0)$.

space component of pinned cyclotron orbits appears in addition to regular skipping and chaotic trajectories. Hence, the phase-space sum rule for $v_{\text {ch }}$ must be modified and Eq. (17) is not valid. In contrast, Eq. (36) still applies, but now we have $\phi^{\prime}=0$ for all $\phi$ since no chaotic trajectory reaches the upper wall. Therefore we have

$$
\begin{aligned}
\langle\Delta x\rangle_{\phi} & =2 r\langle\sin \phi\rangle_{\phi} \\
& =2 r \frac{1}{2} \int_{-1}^{+1} \mathrm{~d} \cos \phi \sin \phi \\
& =\frac{\pi}{2} r \\
\langle\Delta l\rangle_{\phi} & =2 r\langle\phi\rangle_{\phi} \\
& =\pi r
\end{aligned}
$$

and finally

$$
v_{\mathrm{ch}}=\frac{v_{0}}{2} .
$$

\subsection{Diffusion constant}

Using the method of the previous subsection we can also calculate the diffusion constant $D$ of our simplified model. Starting point is the well-known Green-Kubo relation expressing $D$ as integral over the velocity autocorrelation function,

$$
D=\int_{-\infty}^{+\infty} \mathrm{d} t\left\langle\tilde{v}_{x}(t) \tilde{v}_{x}(0)\right\rangle .
$$

Here $\tilde{v}_{x}(t)=v_{0} \cos \varphi(t)-v_{\mathrm{ch}}$ denotes the fluctuation of the transport velocity around its mean value and the average $\langle\ldots\rangle$ is taken over all possible trajectories. For the moment we will assume $\alpha=0$, i.e., no correlations between different segments of a trajectory. In 
this case it suffices to restrict the integral over $t$ to the segment containing the phasespace point $\left(\phi_{0}, l_{0}\right)$ at $t=0$, that is to the interval $-l_{0} \leq v_{0} t \leq \Delta l\left(\phi_{0}\right)-l_{0}$. We use Eq. (34) to average over $\left(\phi_{0}, l_{0}\right)$, make the substitution $l=l_{0}+v_{0} t$ and apply Eq. (35). In this way we find

$$
\begin{aligned}
D_{\alpha=0}= & \frac{1}{\langle\Delta l\rangle_{\phi}} \int_{0}^{\pi} \mathrm{d} \phi_{0} P\left(\phi_{0}\right) \int_{0}^{\Delta l\left(\phi_{0}\right)} \mathrm{d} l_{0} \mathrm{~d} l v_{0}^{-1} \\
& \times\left(v_{0} \cos \varphi\left(\phi_{0}, l\right)-v_{\mathrm{ch}}\right)\left(v_{0} \cos \left(\phi_{0}, l_{0}\right)-v_{\mathrm{ch}}\right) \\
= & \frac{v_{0}}{\langle\Delta l\rangle_{\phi}} \int_{0}^{\pi} \mathrm{d} \phi_{0} P\left(\phi_{0}\right)\left(\Delta x\left(\phi_{0}\right)-\frac{v_{\mathrm{ch}}}{v_{0}} \Delta l\left(\phi_{0}\right)\right)^{2} \\
= & v_{0} \frac{\left\langle\left[\Delta x-\left(v_{\mathrm{ch}} / v_{0}\right) \Delta l\right]^{2}\right\rangle_{\phi}}{\langle\Delta l\rangle_{\phi}} .
\end{aligned}
$$

This result reduces the calculation of the diffusion constant to a simple 1D quadrature which is numerically straightforward. An explicit result is available for strong magnetic field

$$
D_{\alpha=0}=\frac{2 v_{0} r}{3 \pi} \quad\left(r \leq \frac{1}{2}\right),
$$

which reproduces the initially linear dependence of the diffusion constant on the cyclotron radius (Fig. 9).

If $\alpha \neq 0$, several consecutive segments of a trajectory can be identical. Accordingly, the section of the trajectory which is correlated with the point $\left(\phi_{0}, l_{0}\right)$ is longer and

the integration over $l$ in Eq. (45) extends over the range $-l_{0}-\mu \Delta l\left(\phi_{0}\right) \leq l \leq$ $\Delta l\left(\phi_{0}\right)-l_{0}+\nu \Delta l\left(\phi_{0}\right)$ where $\mu, \nu \geq 0$ count the identical segments before and after the current one. Hence the integral increases by a factor $\mu+\nu+1$. For $\nu$ identical segments following the current one we need $\nu$ specular reflections from the lower wall and finally one random reflection. The probability for this is $\alpha^{\nu}(1-\alpha)$. Similarly, the probability for $\mu$ identical segments preceeding the current one is $\alpha^{\mu}(1-\alpha)$. Averaging with these probabilities over $\mu, \nu$ yields

$$
\begin{aligned}
D_{\alpha} & =\sum_{\mu, \nu=0}^{\infty} \alpha^{\mu+\nu}(1-\alpha)^{2}(\mu+\nu+1) D_{0} \\
& =\frac{1+\alpha}{1-\alpha} D_{0} .
\end{aligned}
$$

In Fig. 10 we verify these results on the chaotic diffusion constant numerically.

\section{Summary and discussion}

In this paper we have introduced and studied a particular billiard model which shows directed chaos. The most appealing feature of this model is the fact that the phase space has, to a good approximation, a very simple structure which can be understood both intuitively and analytically. It consists of two invariant manifolds, one chaotic and one regular. The latter is formed by trajectories skipping periodically along one perfectly straight wall of a channel. The other wall has semicircular obstacles or is disordered 

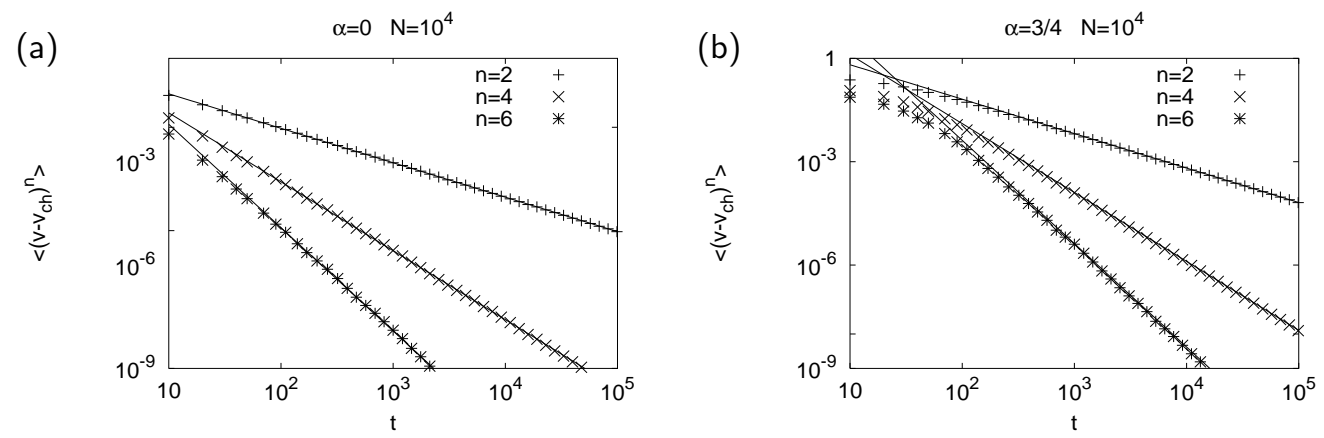

Figure 10. Some higher moments of the distribution of $N=10^{4}$ time-averaged chaotic transport velocities in the magnetic billiard with rough lower wall and $r=2 b$. The probability of specular reflection is (a) $\alpha=0$ and (b) $\alpha=3 / 4$. Straight lines indicate diffusive spreading with the diffusion constant given by Eqs. (46) and (48). The latter correctly predicts that $D_{\mathrm{ch}}$ is greater by a factor 7 in (b).

or rough. This leads to strong back scattering and thus to a chaotic or random phasespace component. Both, the particular phase-space structure and the fact that timereversal symmetry is broken, are dependent on the presence of a transversal magnetic field acting on charged particles such as electrons. The magnetic field should have a suitable intermediate strength. For very small magnetic field the phase-space volume of the regular skipping trajectories goes to zero and, accordingly, the chaotic transport vanishes. For strong magnetic field the phase-space structure changes as more and more regular regions appear. Finally, for very strong magnetic field, pinned cyclotron orbits cover most of the phase space. In this regime the chaotic trajectories are skipping along the lower wall and $v_{\mathrm{ch}}$ approaches a non-zero constant, but at the same time the chaotic fraction of phase space goes to zero.

We have stressed in the introduction the analogies between directed chaos in driven systems and in magnetic billiards. However, there exists also an important difference. It has been shown that driven Hamiltonian systems with directed chaos can generate directed transport in ensembles of particles which have initially a thermal distribution in phase space 1, 2, 3, 4, 5, 6. They do so without an external bias force and under the influence of a potential which is periodic both in space and in time. This is very similar to the concept of stochastic ratchets (Brownian motors) [13, 14, 15, 16. Hence the term Hamiltonian ratchet is appropriate for the models investigated in [1, 2, 3, 4, 5] and also for the atom-optics setup studied experimentally in [6]. However, magnetic billiards with directed chaos are no Hamiltonian ratchets and cannot generate directed transport from thermal ensembles. The reason is the conservation of energy. We have seen that the total transport from all regular and chaotic phase-space components of an energy shell must vanish. Therefore directed transport in magnetic billiards requires control over the initial conditions beyond prescribing a certain distribution of energy values. For example, electrons could be placed selectively into the chaotic phase-space component of our billiard chain if they enter the system from a lead which is attached to the lower wall of the waveguide. 
An interesting extension of the present work would be the inclusion of quantum effects. Definitely they play an important role when applications to electronic transport in semiconductor nanostructures are the goal. For a given geometry and a given time there is always a semiclassical regime in which the effective value of Planck's constant is sufficiently small such that the classical results obtained above remain valid. On the other hand, for a given finite $\hbar$ there should be interesting deviations from our predictions for large times when tunneling between the regular and the chaotic regions of phase space may become important and when the presence or absence of disorder in the system is crucial.

\section{Acknowledgments}

We profitted from discussions with $\mathrm{T}$. Dittrich and from financial support of the Volkswagen Foundation (contract I/78235).

\section{References}

[1] S. Flach, O. Yevtushenko, and Y. Zolotaryuk. Phys. Rev. Lett., 84:2358, 2000.

[2] H. Schanz et al. Phys. Rev. Lett., 87:070601, 2001.

[3] S. Denisov et al. Physica D, 170:131, 2002.

[4] H. Schanz, T. Dittrich, and R. Ketzmerick. Phys. Rev. E, 71:026228, 2005.

[5] T. S. Monteiro et al. Phys. Rev. Lett., 89:194102, 2002.

[6] P. H. Jones et al. Preprint physics/0504096, 2005.

[7] W. Acevedo and T. Dittrich. Prog. Theor. Phys. Suppl., 150:313, 2003.

[8] K. M. Frahm and D. L. Shepelyansky. Phys. Rev. Lett., 78:1440, 1997.

[9] M. Leadbeater, V. I. Falko, and C. J. Lambert. Phys. Rev. Lett., 81:1274, 1998.

[10] M. Prusty (unpublished).

[11] Ya. G. Sinai. Russian Mathematical Survey, 25:137, 1970.

[12] M. Horvat and T. Prosen. J. Phys. A, 37:3133, 2004.

[13] R. P. Feynman, R. B. Leighton, and M. Sands. The Feynman Lectures on Physics. AddisonWesley, Reading, MA, 1966.

[14] P. Reimann. Phys. Rep., 361:57, 2002.

[15] R. D. Astumian and P. Hänggi. Physics Today, 55(11):33, 2002.

[16] F. Jülicher, A. Ajdari, and J. Prost. Rev. Mod. Phys., 69:1269, 1997. 